Boundary-equilibrium bifurcations in piecewise-smooth slow-fast systems

Kowalczyk, Piotr and Glendinning, Paul

2011

MIMS EPrint: $\mathbf{2 0 1 1 . 2 4}$

Manchester Institute for Mathematical Sciences

School of Mathematics

The University of Manchester

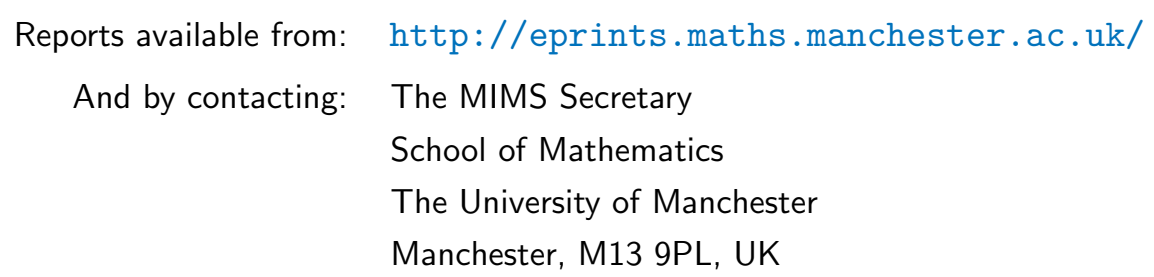

ISSN 1749-9097 


\section{Boundary-equilibrium bifurcations in piecewise-smooth slow-fast systems}

P. Kowalczyk ${ }^{1, \text { a) }}$ and P. Glendinning ${ }^{2, \text { b) }}$

${ }^{1)}$ Centre for Interdisciplinary Computational and Dynamical Analysis (CICADA), University of Manchester, Manchester, M13 9PL, U.K. and Manchester Metropolitan University, School of Computing,

Mathematics and Digital Technology, Manchester, U.K.

${ }^{2)}$ School of Mathematics and Centre for Interdisciplinary Computational and Dynamical Analysis (CICADA), University of Manchester, Manchester, M13 9PL, U.K.

(Dated: 3 March 2011)

In the paper we study the qualitative dynamics of piecewise-smooth slow-fast systems (singularly perturbed systems). We consider phase space topology of systems with 1-dimensional slow dynamics and 1-dimensional fast dynamics. The slow manifold of the reduced system is formed by a piecewise-continuous curve, and the differentiability is lost across the switching surface. In the full system the slow manifold is no longer continuous, and there is an $\mathcal{O}(\varepsilon)$ discontinuity across the switching manifold, but the discontinuity cannot qualitatively alter system dynamics. Revealed phase space topology is used to unfold qualitative dynamics of planar slow-fast systems with an equilibrium point on the switching surface. In this case the local dynamics corresponds to so-called boundary-equilibrium bifurcations, and four qualitative phase portraits are uncovered. Our results are then used to investigate the dynamics of a box model of a thermohaline circulation, and the presence of a boundary-equilibrium bifurcation of a fold type is shown.

The effect of singular perturbations on bifurcations in non-smooth systems, in many instances, is not known. In this paper we study the dynamics of planar slow-fast systems with an equilibrium point on the switching surface. This situation corresponds to singularly perturbed boundary-equilibrium bifurcations. We show that four qualitative different phase portraits exist in the reduced system. Effects of singular perturbations on these phase portraits are studied. Our results are then used to show the presence of a non-smooth equivalent of a fold bifurcation in a box model of a thermohaline circulation.

\section{INTRODUCTION}

Many systems of relevance to applications are modelled using piecewise-smooth dynamical systems. Examples include systems modelled by a set of ordinary differential equations that lose their smoothness properties across co-dimension one manifolds in phase space. Applications include DC/DC power converters which are modelled using distinct sets of ordinary differential equations between which a converter switches depending on whether the switching element is in the on or off state $^{1}$; friction oscillators ${ }^{2-4}$, impacting systems ${ }^{5-7}$ are other examples of relevance to applications which are modelled by systems with discontinuous nonlinearities.

Much research effort has recently been spent on understanding the qualitative dynamics of these systems and a theory of phase space transitions triggered by

\footnotetext{
a)Electronic mail: piotr.kowalczyk@manchester.ac.uk

b) Electronic mail: paul.glendinning@manchester.ac.uk
}

the presence of discontinuous nonlinearities has been developed $^{8-10}$. These phase space transitions are termed discontinuity induced bifurcations, DIBs for short. At present there exists a fairly complete description of codimension one DIBs of limit cycles and equilibrium points. However, one of the pressing issues with regards to investigations of the dynamics of piecewise-smooth systems is the effect of singular perturbations. For sufficiently differentiable vector fields the theory developed by Tikhonov $^{11}$ and Fenichel ${ }^{12}$ makes it possible to treat singular perturbation problems as regular ones by proving the existence of a hyperbolic invariant manifold. This is not the case when piecewise-smooth systems are treated. Due to the presence of the discontinuity a slow manifold usually exhibits a discontinuity across a surface (or hyper-surface) of phase space where the smoothness property of vector fields governing system equations are lost. This has been shown in ${ }^{13}$ for systems with discontinuous vector fields (Filippov systems). The presence of the discontinuity may carry significant implications regarding system dynamics, e.g., it may play a crucial role in the onset of micro-chaotic dynamics ${ }^{14}$. This in turn carries practical implications: seemingly noisy output from an experiment may result from the fast dynamics neglected in the modelling procedure and be in fact of a deterministic nature. The knowledge of the effects of singular perturbations on system dynamics would then allow one to establish the 'size' of this neglected dynamics and provide more insight into the experiment. For these reasons it is necessary to complete the theory of piecewise-smooth systems by considering the effects of singular perturbations.

$\operatorname{In}^{13,19}$ the authors studied the effects of singular perturbations on systems with discontinuous vector fields under the simplifying assumption that the switching decision does not depend on the fast variable, which allowed them to prove that stable periodic orbits persist, but acquire small boundary layers, due to the applied singular 
perturbation. As it was pointed out in ${ }^{14}$, in practise, this assumption may be difficult to satisfy, and hence it does not cover all cases of practical interest. In our paper we assume that the switching depends on the fast variable.

In our work we focus on a class of piecewise-smooth systems which are continuous in the phase space region of interest, but the vector field derivatives are discontinuous across a smooth manifold (switching manifold). We then investigate the case when there is an equilibrium existing on the switching surface in which case we cannot apply Fenichel's theory to study system dynamics.

The paper is organized as follows. In Sec. II we introduce the slow-fast piecewise-smooth systems studied here. The slow and fast dynamics is one-dimensional. In Sec. III phase space topology, and in particular the topology of the slow manifold, of the reduced and full system is investigated. The discontinuity of the slow manifold in the full system is established and the size of the discontinuity is linked with the differentiability properties of the vector fields across the switching manifold. In Sec. IV the qualitative dynamics of the reduced and full system is investigated in the special case that an equilibrium point exists on the switching surface. This is a natural co-dimension one bifurcation point and we unfold four qualitatively distinct phase portraits that can be observed in the full system. Then, in Sec. V a box model of a thermohaline circulation is analyzed. The model is a planar piecewise-smooth slow-fast system obtained by a novel scaling of an example due to ${ }^{15}$. We then use our results from Sec. IV to analyze the dynamics of the model. In particular, the presence of a non-smooth equivalent of a fold bifurcation is explained. Finally in Sec. VI we conclude the paper highlighting open problems and indicating further directions for the theory of singularly perturbed piecewise-smooth systems.

\section{SWITCHED SLOW-FAST SYSTEMS}

Consider slow-fast systems of the form

$$
\begin{gathered}
\dot{y}= \begin{cases}g_{+}(x, y ; \varepsilon) & \text { if } h(x, y) \geq 0, \\
g_{-}(x, y ; \varepsilon) & \text { if } h(x, y)<0,\end{cases} \\
\varepsilon \dot{x}= \begin{cases}f_{+}(x, y ; \varepsilon) & \text { if } h(x, y) \geq 0, \\
f_{-}(x, y ; \varepsilon) & \text { if } h(x, y)<0,\end{cases}
\end{gathered}
$$

where $x \in \mathbb{R}, y \in \mathbb{R}$, and $\varepsilon>0$ is a small parameter measuring the difference in the time scale between the evolution of fast $(x)$ and slow variables $(y)$. Assume that $f_{ \pm}: \mathbb{R}^{2} \times \mathbb{R} \mapsto \mathbb{R}, \mathbf{g}_{ \pm}: \mathbb{R}^{2} \times \mathbb{R} \mapsto \mathbb{R}$ are sufficiently differentiable functions of $x, y$ and $\varepsilon$, well defined for all $(x, y)$ in a region of interest. The zero-level set of function $h: \mathbb{R}^{2} \mapsto \mathbb{R}$ defines the switching surface, $\Sigma$, i.e.

$$
\Sigma:=\left\{(x, y) \in \mathbb{R}^{2}: h(x, y)=0\right\}
$$

with $h(x, y)=0$ being a regular value. Note that $h$ is independent of $\varepsilon$. We assume that $f_{-}=f_{+}$and $g_{-}=g_{+}$ for $(x, y) \in \Sigma$, but $\nabla f_{+} \neq \nabla f_{-}$and $\nabla g_{+} \neq \nabla g_{-}$on $\Sigma$, where ' $\nabla$ ' denotes the gradient differential operator. In what follows we will also use the notation $\nabla f_{ \pm}=$ $\left[f_{ \pm x}, f_{ \pm y}\right]$ and $\nabla g_{ \pm}=\left[g_{ \pm x}, g_{ \pm y}\right]$, where subscripts ' $x$ ' and ' $y$ ' denote partial differentiation with respect to ' $x$ ' and ' $y$ ' variables.

Furthermore, define regions where the system dynamics is smooth and governed by the slow-fast systems $\left(f_{+}, g_{+}\right)$and $\left(f_{-}, g_{-}\right)$respectively, as

$$
\begin{aligned}
& G_{+}:=\left\{(x, y) \in \mathbb{R}^{2}: h(x, y)>0\right\}, \\
& G_{-}:=\left\{(x, y) \in \mathbb{R}^{2}: h(x, y)<0\right\} .
\end{aligned}
$$

We assume further that the nonlinear equations $f_{ \pm}(x, y ; 0)=0$ can be solved for $x$ for all $y$ giving $x_{ \pm}^{0}(y)$ respectively. We also assume the stability condition

$$
0>-c>f_{ \pm x}\left(x_{ \pm}^{0}(y), y ; 0\right), \quad \forall y
$$

where $c$ is a positive constant. Condition (3) is the standard condition for the application of Fenichel Theory ${ }^{12}$.

\section{PHASE SPACE TOPOLOGY}

\section{A. General description}

Let us first consider phase space topology of (1) and (2) when $\varepsilon=0$, that is, we start by considering the reduced system. Relation $f_{+}(x, y, 0)=0$ defines a smooth manifold, say $\mathcal{M}_{+}$, in $G_{+}$, and correspondingly $f_{-}(x, y, 0)=0$ defines a manifold, say $\mathcal{M}_{-}$, in $G_{-}$. In what follows we use the following notation $f_{ \pm}^{0}\left(x^{0}(y), y\right)=f_{ \pm}^{0}\left(x^{0}(y), y ; 0\right)=f_{ \pm}^{0}(x, y)$. Let $\left(x\left(y_{-}^{*}\right), y_{-}^{*}\right)$ and $\left(x\left(y_{+}^{*}\right), y_{+}^{*}\right)$ be the points of intersection of $\mathcal{M}_{-}$and $\mathcal{M}_{+}$with $\Sigma$ respectively. Assume that $\mathcal{M}_{ \pm}$ are transversal to $\Sigma$ at $\left(x^{0}\left(y_{-}^{*}\right), y_{-}^{*}\right)=\left(x^{0}\left(y_{+}^{*}\right), y_{+}^{*}\right)$, i.e., $\left\langle h_{T}, \nabla f_{ \pm}^{0}\right\rangle \neq 0$ on $\Sigma$, where $h_{T}$ is a vector tangent to $\Sigma$, and $\nabla f_{ \pm}^{0}$ is a vector normal to $\left\{f_{ \pm}^{0}\left(x^{0}(y), y\right)=0\right\}$, and $\langle$,$\rangle denotes the dot product. The transversality can be$ assumed without loss of generality. By assumption, on $\Sigma$ we have $f_{+}^{0}\left(x^{0}(y), y\right)=f_{-}^{0}\left(x^{0}(y), y\right)=0$, and the slow manifold of the reduced system, namely $\mathcal{M}_{+} \cup \mathcal{M}_{-}$, is continuous across $\Sigma$.

In this setting we define the reduced system that lives on $\mathcal{M}_{-} \cup \mathcal{M}_{+}$as

$$
\dot{y}= \begin{cases}g_{+}\left(x_{+}^{0}(y), y ; 0\right) & \text { if } h(x, y) \geq 0 \\ g_{-}\left(x_{-}^{0}(y), y ; 0\right) & \text { if } h(x, y)<0\end{cases}
$$

Clearly, the reduced system (4) evolves on a continuous and piecewise-smooth slow manifold $\mathcal{M}=\mathcal{M}_{+} \cup \mathcal{M}_{-}$. Consider now how the structure of phase space changes for $\varepsilon>0$. 
In each of the regions $G_{+}$and $G_{-}$there exist invariant manifolds, say $\mathcal{M}_{+}^{\varepsilon}$ and $\mathcal{M}_{-}^{\varepsilon}$ respectively. These manifolds need not be unique and by the continuity argument they are within $\mathcal{O}(\varepsilon)$ distance from $\mathcal{M}_{+}$and $\mathcal{M}_{-}$. It is important to determine if these manifolds can create a continuous manifold, say $\mathcal{M}^{\varepsilon}=\mathcal{M}_{+}^{\varepsilon} \cup \mathcal{M}_{-}^{\varepsilon}$ across $\Sigma$.

We assume that the slow manifold $\mathcal{M}_{+}^{\varepsilon}$ can be approximated by the asymptotic series in $\varepsilon$, i.e.

$$
x_{+}(y)=\alpha_{0}(y)+\varepsilon \alpha_{1}(y)+\mathcal{O}\left(\varepsilon^{2}\right),
$$

and its coefficients are such that (5) solves (2) for $\varepsilon>0$; obviously $\alpha_{0}(y), \alpha_{1}(y), \cdots$, are coefficients (functions of the slow variable $y$ ) to be determined.

The slow manifold $\mathcal{M}_{-}^{\varepsilon}$ can then be given by an equivalent approximate expression

$$
x_{-}(y)=\beta_{0}(y)+\varepsilon \beta_{1}(y)+\mathcal{O}\left(\varepsilon^{2}\right),
$$

where again $\beta_{0}(y), \beta_{1}(y), \cdots$, are coefficients (functions of the slow variable $y$ ) to be determined. Let us express $f_{ \pm}(x, y ; \varepsilon)$ and $g_{ \pm}(x, y ; \varepsilon)$ as power series in $\varepsilon$. We then have

$$
f_{ \pm}(x, y ; \varepsilon)=f_{ \pm}^{(0)}(x, y)+\varepsilon f_{ \pm}^{(1)}(x, y)+\mathcal{O}\left(\varepsilon^{2}\right)
$$

and

$$
g_{ \pm}(x, y ; \varepsilon)=g_{ \pm}^{(0)}(x, y)+\varepsilon g_{ \pm}^{(1)}(x, y)+\mathcal{O}\left(\varepsilon^{2}\right) .
$$

We now follow the standard procedure and use (1), (2), and (5)-(8) to determine $\alpha_{0}, \alpha_{1}, \beta_{0}$, and $\beta_{1}$. Obviously $\alpha_{0} \equiv x_{+}^{0}(y)$ and $\beta_{0} \equiv x_{-}^{0}(y)$.

We also have $\frac{d}{d y}\left(f_{+}^{(0)}\left(\alpha_{0}(y), y\right)\right)=0$.

Hence $f_{+x}^{(0)}\left(\alpha_{0}, y\right) \alpha_{0}^{\prime}(y)+f_{+y}^{(0)}\left(\alpha_{0}, y\right)=0$, which gives

$$
\alpha_{0}^{\prime}(y)=-\frac{f_{+y}^{(0)}}{f_{+x}^{(0)}},
$$

and is well defined provided that $f_{+x}^{(0)} \neq 0$, and similarly

$$
\beta_{0}^{\prime}(y)=-\frac{f_{-y}^{(0)}}{f_{-x}^{(0)}}
$$

and is well defined provided that $f_{-x}^{(0)} \neq 0$. It then follows that

$$
\alpha_{0}^{\prime}(y) \neq \beta_{0}^{\prime}(y) \Leftrightarrow f_{+x}^{0} f_{-y}^{0}-f_{+y}^{0} f_{-x}^{0} \neq 0 .
$$

Using standard procedure we now compute terms of $\mathcal{O}(\varepsilon)$ which yields

$$
\alpha_{1}(y)=\frac{-1}{\left[f_{+x}^{(0)}\right]^{2}}\left(f_{+y}^{(0)} g_{+}^{(0)}+f_{+}^{(1)} f_{+x}^{(0)}\right)
$$

with the quantities on the right-hand side of (12) evaluated at $\left(\alpha_{0}(y), y\right)$, and $\beta_{1}(y)$

$$
\beta_{1}(y)=\frac{-1}{\left[f_{-x}^{(0)}\right]^{2}}\left(f_{-y}^{(0)} g_{-}^{(0)}+f_{-}^{(1)} f_{-x}^{(0)}\right)
$$

with the quantities on the right-hand side of (13) evaluated at $\left(\beta_{0}(y), y\right)$. We now wish to determine if $x_{+}(y)$ and $x_{-}(y)$ are continuous on $\Sigma$.

Suppose that $\left(x_{-}\left(y_{-}^{i n}\right), y_{-}^{i n}\right)$ and $\left(x_{+}\left(y_{+}^{i n}\right), y_{+}^{i n}\right)$ are the points of intersection of $\mathcal{M}_{-}^{\varepsilon}$ and $\mathcal{M}_{+}^{\varepsilon}$ with $\Sigma$ respectively, and that $\Sigma$ is defined by the equation $h(x, y)=0$, with no $\varepsilon$ dependence (although such dependence could easily be treated). Recall that $\mathcal{M}_{-}$and $\mathcal{M}_{+}$intersect $\Sigma$ at the same point $\left(x^{0}\left(y^{*}\right), y^{*}\right)$. Thus we are looking to find the $\mathcal{O}(\varepsilon)$ terms for the intersection points, and we can expand

$$
y_{ \pm}^{i n}=y^{*}+\varepsilon y_{ \pm 1}^{*}+\mathcal{O}\left(\varepsilon^{2}\right),
$$

and seek to find $y_{ \pm 1}^{*}$ and the corresponding correction to the $x$-coordinates.

By definition

$$
\begin{aligned}
x_{+}\left(y_{+}^{i n}\right) & =\alpha_{0}\left(y_{+}^{i n}\right)+\alpha_{1}\left(y_{+}^{i n}\right) \varepsilon+\mathcal{O}\left(\varepsilon^{2}\right) \\
& =\alpha_{0}\left(y^{*}+\varepsilon y_{ \pm 1}^{*}\right)+\alpha_{1}\left(y^{*}\right) \varepsilon+\mathcal{O}\left(\varepsilon^{2}\right) \\
& =\alpha_{0}\left(y^{*}\right)+\left(\alpha_{0}^{\prime}\left(y^{*}\right) y_{ \pm 1}^{*}+\alpha_{1}\left(y^{*}\right)\right) \varepsilon+\mathcal{O}\left(\varepsilon^{2}\right)
\end{aligned}
$$

and so $h\left(x_{+}\left(y_{+}^{i n}\right),\left(y_{+}^{i n}\right)\right)=0$ becomes (retaining only terms up to order $\varepsilon$ )

$$
h\left(x^{*}, y^{*}\right)+\left(h_{x}^{*}\left(\alpha_{0}^{\prime}\left(y^{*}\right) y_{+1}^{*}+\alpha_{1}\left(y^{*}\right)\right)+h_{y}^{*} y_{+1}^{*}\right) \varepsilon=0
$$

where starred functions are evaluated at $\left(\alpha_{0}\left(y^{*}\right), y^{*}\right)$. Using (16) we have that

$$
\left(\alpha_{0}^{\prime}\left(y^{*}\right)+h_{y} / h_{x}\right) y_{+1}^{*}=-\alpha_{1}\left(y^{*}\right) .
$$

Using (17) we then have that

$$
x_{+}\left(y_{+}^{i n}\right)=\alpha_{0}\left(y^{*}\right)-\frac{1}{h_{x}} h_{y} y_{+1}^{*} \varepsilon .
$$

Similarly, we have that

$$
x_{-}\left(y_{-}^{i n}\right)=\beta_{0}\left(y^{*}\right)-\frac{1}{h_{x}} h_{y} y_{-1}^{*} \varepsilon .
$$

We should note here that $h_{x} \neq 0$ by our initial assumption that the switching function depends on the fast variable.

Let us now express $h_{y} / h_{x}$ using the continuity condition of the vector field $f$ across the switching surface $\Sigma$. If the boundary $\Sigma$ is written in the form $x=b(y)$ then $h(b(y), y)=0$ implies that $b^{\prime}(y)=-h_{y} / h_{x}$ by the same argument which showed (9). But since $f$ is continuous across $\Sigma$ then $f_{+}^{(0)}(b(y), y)-f_{-}^{(0)}(b(y), y)=$ 0 and so the same argument implies that $b^{\prime}(y)=$ $-\left(f_{+y}^{(0)}-f_{-y}^{(0)}\right) /\left(f_{+x}^{(0)}-f_{-x}^{(0)}\right)$ on the boundary, and so putting these two equations for $b^{\prime}(y)$ together we obtain

$$
\frac{h_{y}}{h_{x}}=\frac{\left(f_{+y}^{(0)}-f_{-y}^{(0)}\right)}{\left(f_{+x}^{(0)}-f_{-x}^{(0)}\right)} .
$$

Now, the first term in (16) is zero by the definition of $y^{*}$, and so setting the $\varepsilon$ term to zero gives

$$
y_{+1}^{*}=-\frac{h_{x}^{*} \alpha_{1}\left(y^{*}\right)}{h_{x}^{*} \alpha_{0}^{\prime}\left(y^{*}\right)+h_{y}}
$$


provided $h_{x} \alpha_{0}^{\prime}\left(y^{*}\right)+h_{y} \neq 0$, or, using (9),

$$
y_{+1}^{*}=-\frac{h_{x}^{*} f_{+x}^{(0) *} \alpha_{1}\left(y^{*}\right)}{h_{y}^{*} f_{+x}^{(0) *}-h_{x}^{*} f_{+y}^{(0) *}}=-\frac{f_{+x}^{(0) *} \alpha_{1}\left(y^{*}\right)}{\frac{h_{y}^{*}}{h_{x}^{*}} f_{+x}^{(0) *}-f_{+y}^{(0) *}}
$$

with $\alpha_{1}$ given by (12). Replacing the ratio $h_{y} / h_{x}$ in the lowest order expansion by the right hand side of (18) evaluated at $y^{*}$ after a little tidying up, using (12), gives

$$
y_{+1}^{*}=\frac{\left(f_{+x}^{(0)}-f_{-x}^{(0)}\right)\left(g_{+}^{(0)} f_{+y}^{(0)}+f_{+}^{(1)} f_{+x}^{(0)}\right)}{f_{+x}^{(0)}\left(f_{-x}^{(0)} f_{+y}^{(0)}-f_{-y}^{(0)} f_{+x}^{(0)}\right)} .
$$

A precisely analogous argument yields

$$
y_{-1}^{*}=\frac{\left(f_{+x}^{(0)}-f_{-x}^{(0)}\right)\left(g_{-}^{(0)} f_{-y}^{(0)}+f_{-}^{(1)} f_{-x}^{(0)}\right)}{f_{-x}^{(0)}\left(f_{-x}^{(0)} f_{+y}^{(0)}-f_{-y}^{(0)} f_{+x}^{(0)}\right)} .
$$

Thus $y_{+1}^{*} \neq y_{-1}^{*}$ in general, so $\mathcal{M}^{\varepsilon}$ is discontinuous across $\Sigma$ and the size of the discontinuity is $\mathcal{O}(\varepsilon)$. Note that $x_{+}\left(y_{+}^{i n}\right)$ can be calculated from (21) and (15), with an analogous result for $x_{-}\left(y_{-}^{i n}\right)$.

\section{B. A local co-ordinate system}

In Sec. IV A we consider the dynamics close to the switching surface and near the slow manifolds. To make the analysis as simple as possible it is natural to introduce a co-ordinate set in which the switching surface is given by $\{x=0\}$ in terms of a new $x$ variable. If $\left(x^{*}, y^{*}\right)$ is the intersection of the slow manifolds for $\varepsilon=0$ with the switching surface $\Sigma$ then we assume that the equation

$$
X=h(x, y)
$$

can be inverted near $\left(x^{*}, y^{*}\right)$ giving $x=H(X, y)$. This is possible provided $h_{x}(x, y) \neq 0$ in a neighbourhood of $\left(x^{*}, y^{*}\right)$. Using (1) and (2) we find

$$
\varepsilon \dot{X}=h_{x} f_{ \pm}+\varepsilon h_{y} g_{ \pm}
$$

with the right hand side evaluated at $(H(X, y), y)$, and where the switching surface is now the set $\{X=0\}$.

Defining $Y=y-y^{*}$ we obtain a new set of equations for which the intersection of the slow manifolds when $\varepsilon=0$ is at $(X, Y)=(0,0)$ and the switching surface has $X=0$.

Without writing the new system in full detail we note that in these new coordinates the size of the discontinuity is again $\mathcal{O}(\varepsilon)$ for typical systems.

\section{Special case}

The box model of thermohaline circulation that we study in Sec. $\mathrm{V}$ is a special case of the planar slowfast systems studied here; the function $f(x, y ; 0)$ is at least $C^{1}$ differentiable $\forall(x, y)$ which implies that the slow manifold of the reduced system is a smooth curve across $\Sigma$. Thus, we will consider how the smoothness of the slow manifold affects system dynamics. Let us assume that the fast dynamics is given by

$$
\varepsilon \dot{x}=f^{(0)}(x, y)+ \begin{cases}\varepsilon f_{+}^{(1)}(x, y)+\mathcal{O}\left(\varepsilon^{2}\right) & \text { if } h(x, y) \geq 0 \\ \varepsilon f_{-}^{(1)}(x, y)+\mathcal{O}\left(\varepsilon^{2}\right) & \text { if } h(x, y)<0\end{cases}
$$

where $f_{+}^{(1)}=f_{-}^{(1)}$ for $(x, y)$ on $\Sigma$ but $\nabla f_{+}^{(1)} \neq \nabla f_{-}^{(1)}$ (by the assumption on the continuity of the vector fields across the switching manifold the coefficients at all orders of $\varepsilon$ are equal on $\Sigma$ ). In this setting, when we consider the reduced system, we note that the equation $f^{(0)}(x, y)=0$ defines the slow manifold of the reduced system $\mathcal{M}=$ $\mathcal{M}_{+} \cup \mathcal{M}_{-}$, and $\mathcal{M}$ is a smooth curve across $\Sigma$ by the fact that $f^{(0)}(x, y)$ is differentiable; obviously $\alpha_{0}(y) \equiv$ $x_{+}^{0}(y) \equiv \beta_{0}(y) \equiv x_{-}^{0}(y)$.

It might now appear from (21) and (22) that $y_{+1}^{*}=$ $y_{-1 *}$, but a closer look at the derivation of (18) reveals that this relation uses $\nabla f_{+}^{(0)} \neq \nabla f_{-}^{(0)}$. This does not hold here, and so the correct leading order relation is

$$
\frac{h_{y}}{h_{x}}=\frac{\left(f_{+y}^{(1)}-f_{-y}^{(1)}\right)}{\left(f_{+x}^{(1)}-f_{-x}^{(1)}\right)}
$$

which can be substituted into (20) to find a new expression for $y_{+1}^{*}$, with an analogous argument giving a revised expression for $y_{-1}^{*}$. In general there is no reason for these to be equal and hence in general we expect the splitting of the manifolds to be first order in $\varepsilon$.

Indeed, this argument shows that even if the splitting in $f$ is $\mathcal{O}\left(\varepsilon^{N}\right), N \geq 2$, the slow manifolds are separated by $\mathcal{O}(\varepsilon)$ on $\Sigma$.

\section{QUALITATIVE DYNAMICS}

In this section we will consider the dynamics of slowfast system (1) and (2) at the point of intersection of the slow manifold $\mathcal{M}$ of the reduced system with the switching surface. Away from this point Fenichel's theory holds and standard results can be applied to study system dynamics. Let us assume that the switching surface is given by $\Sigma:=\{h(x, y)=x=0\}$, and the slow manifold $\mathcal{M}$ of the reduced system crosses $\Sigma$ at the origin as described in Section III B.

\section{A. Case I: No-equilibrium of the reduced system on the switching surface}

We will begin by expanding $f_{ \pm}$about the origin and use this to unfold the dynamics of the full slow-fast system around the origin. Thus, the slow system which we 
shall study is left unchanged except for the switching surface, i.e. we have

$$
\dot{y}= \begin{cases}g_{+}(x, y ; \varepsilon) & \text { if } x \geq 0 \\ g_{-}(x, y ; \varepsilon) & \text { if } x<0\end{cases}
$$

and the fast system becomes

$$
\varepsilon \dot{x}= \begin{cases}-C_{+} x+B y+\varepsilon D+\mathcal{O}\left(\|x, y, \varepsilon\|^{2}\right) & \text { if } x \geq 0 \\ -C_{-} x+B y+\varepsilon D+\mathcal{O}\left(\|x, y, \varepsilon\|^{2}\right) & \text { if } x<0\end{cases}
$$

where $C_{+}>0$ and $C_{-}>0$ and we can choose $B$ to be positive or negative in the subsequent qualitative analysis. Without loss of generality we choose to consider positive $B$ (negative $B$ implies change of the direction of the flow on the slow manifold of the reduced system but the subsequent analysis can be conducted in like manner).

The form of the fast subsystem is the result of the continuity across $\Sigma$ and the sign of $C_{+}$and $C_{-}$being positive is determined by the fact that we consider singular perturbations (see Eq. 3), that is, all trajectories away from the slow manifold approach it exponentially fast. Thus setting $\varepsilon=0$ in (28) gives the local approximation for the slow manifold of the reduced system as the union of $\mathcal{M}_{+}:=\left\{x_{1}=B C_{+}^{-1} y\right\}$ and $\mathcal{M}_{-}:=\left\{x_{2}=B C_{-}^{-1} y\right\}$. Note that both $\mathcal{M}_{+}$and $\mathcal{M}_{-}$exist in $G_{+} \cup \Sigma \cup G_{-}$but we are only considering these parts which pertain to their domains of definition. Clearly $\mathcal{M}$ locally around the origin is a piecewise linear manifold. Let us assume that at the origin $\dot{y}(0)>0$. Then on the slow manifold $\mathcal{M}$, $\dot{x}\left(0_{-}\right)=B C_{-}^{-1} \dot{y}(0)>0$ and $\dot{x}\left(0_{+}\right)=B C_{+}^{-1} \dot{y}(0)>0$. Thus in the reduced system, locally around the origin the trajectory crossing $\Sigma$ exhibits a corner along its evolution and moves on $\mathcal{M}$ towards increasing values of $x$ and $y$. Note that considering negative $B$ in (28) implies the evolution to the left across $\Sigma$ along the decreasing values of $x$.

Let us now consider the dynamics of the full system locally around the origin. Assume $C_{+}>C_{-}>0$. Then $B / C_{-}>B / C_{+}>0$. Since the reduced system does not have an equilibrium at the origin then $g_{ \pm}(0,0 ; 0)=a>0$ (we assume without loss of generality $a$ to be positive). For sufficiently small $\varepsilon$, by the continuity argument, $g_{ \pm}(0,0, \varepsilon) \approx a>0$. Let us determine approximate expressions that define slow manifolds $\mathcal{M}_{+}^{\varepsilon}$ and $\mathcal{M}_{-}^{\varepsilon}$ about the origin. We use the power series expansions as explained in the former section. Thus, $\mathcal{M}_{ \pm}^{\varepsilon}$ are approximately defined by the relations $x_{+}(y)=\alpha_{0}+\varepsilon \alpha_{1}$ and $x_{-}(y)=\beta_{0}+\varepsilon \beta_{1}$ respectively, where $\alpha_{0}, \alpha_{1}, \beta_{0}, \beta_{1}$ are coefficients to be determined. The coefficients $\alpha_{0}$ and $\beta_{0}$ are obviously the functional expressions for the slow manifolds of the reduced system, and are given by $\alpha_{0}=B C_{+}^{-1} y$ and $\beta_{0}=B C_{-}^{-1} y$ respectively.

We use (12) and (13) to find $\alpha_{1}$ and $\beta_{1}$ respectively. We have $f_{+x}^{(0)}=-C_{+}, f_{+y}^{(0)}=B, f_{+}^{(1)}=D f_{-x}^{(0)}=-C_{-}$, $f_{-y}^{(0)}=B, f_{-}^{(1)}=D$ and $g_{ \pm}^{(0)}=a$. We then obtain $\alpha_{1}=$
$-\left(B C_{+}^{-2} a-D C_{+}^{-1}\right) \varepsilon, \beta_{1}=-\left(B C_{-}^{-2} a-D C_{-}^{-1}\right) \varepsilon$, and the approximate expressions for the slow manifolds can be given by

$$
x_{+}(y)=B C_{+}^{-1} y-B C_{+}^{-2} a \varepsilon+D C_{+}^{-1} \varepsilon,
$$

and

$$
x_{-}(y)=B C_{-}^{-1} y-B C_{-}^{-2} a \varepsilon+D C_{-}^{-1} \varepsilon .
$$

The slow manifold $\mathcal{M}_{+}^{\varepsilon}$ crosses $\Sigma$ at $\left(C_{+}^{-1} a+B^{-1} D\right) \varepsilon$, and the slow manifold $\mathcal{M}_{-}^{\varepsilon}$ crosses $\Sigma$ at $\left(C_{-}^{-1} a+B^{-1} D\right) \varepsilon$. Note that $C_{-}^{-1} a \varepsilon>C_{+}^{-1} a \varepsilon>0$. Thus $\mathcal{M}_{+}$crosses the switching surface below $\mathcal{M}_{-}$and the direction of the flow at the intersection points of $\mathcal{M}_{ \pm}$with $\Sigma$ are $\dot{x}\left(y_{+}\right)=$ $B C_{+}^{-1} a>0$ and $\dot{x}\left(y_{-}\right)=B C_{-}^{-1} a>0$ respectively.

\section{B. Case II: Equilibrium of the reduced system on the switching surface}

Consider now the situation when $g_{ \pm}(0,0,0)=0$, that is, the reduced system exhibits an equilibrium at the origin. We want to determine what is the dynamics of the slow-fast system (27) and (28) around the origin in this case. Before we consider the local dynamics about the origin we need to check whether the general conclusions on the size of the discontinuity of the slow manifold $\mathcal{M}_{+}^{\varepsilon} \cup \mathcal{M}_{-}^{\varepsilon}$ derived in the previous section still hold. Note that (21) and (22) hold when $g_{ \pm}^{(0)}=0$, so

$$
y_{ \pm 1}^{*}=\frac{f_{ \pm}^{(1)}\left(f_{+x}^{(0)}-f_{-x}^{(0)}\right)}{\left(f_{-x}^{(0)} f_{+y}^{(0)}-f_{-y}^{(0)} f_{+x}^{(0)}\right)}
$$

with the functions on the right hand side evaluated at $\left(x\left(y^{*}\right), y^{*}\right)$. But since $f_{-}^{(1)} \neq f_{+}^{(1)}$ in general (the order $\varepsilon$ equation for the continuity of $f$ across the boundary $\Sigma$ contains terms from $f_{ \pm}^{(0)}$ ) so the discontinuity of $\mathcal{M}_{+}^{\varepsilon} \cup$ $\mathcal{M}_{+}^{\varepsilon}$ on $\Sigma$ remains of order $\varepsilon$.

We will now proceed to study system dynamics. Expanding (27) in $x, y$ and $\varepsilon$ to leading order yields

$$
\dot{y}= \begin{cases}c_{+} x+b y+d \varepsilon+\mathcal{O}\left(\|x, y, \varepsilon\|^{2}\right) & x \geq 0, \\ c_{-} x+b y+d \varepsilon+\mathcal{O}\left(\|x, y, \varepsilon\|^{2}\right) & \text { if } x<0 .\end{cases}
$$

In the current case the dynamics of the reduced system (28) and (32) about the origin is characterized by four qualitatively distinct phase portraits. The slow manifolds of the reduced system are given by the same functional expressions as in the previous case, namely $x_{ \pm}=B C_{ \pm}^{-1} y$. Thus the dynamics of the reduced system about the origin will depend on the signs of $\dot{x}$, and $\dot{y}$, for $x$ and $y$ about the origin. About the origin on the slow manifolds $\dot{x}=B C_{ \pm}^{-1}\left(c_{ \pm} B C_{ \pm}^{-1}+b\right) y$ and $\dot{y}=\left(c_{ \pm} B C_{ \pm}^{-1}+b\right) y$. Clearly the aforementioned four cases depend on the signs of $\left(c_{ \pm} B C_{ \pm}^{-1}+b\right)$. 
- Case I, $c_{+} B C_{+}^{-1}+b>0$ and $c_{-} B C_{-}^{-1}+b>0$. The origin is a repeller and for $x>0$ the trajectory moves to the right, and for $x<0$ the trajectory evolves to the left, away from the origin (See Fig. 1 (a)).

- Case II, $c_{+} B C_{+}^{-1}+b>0$ and $c_{-} B C_{-}^{-1}+b<0$. The origin is a saddle and for $x>0$ the trajectory moves away from the origin, and for $x<0$ the trajectory evolves toward the origin (See Fig. 1 (b)).

- Case III, $c_{+} B C_{+}^{-1}+b<0$ and $c_{-} B C_{-}^{-1}+b<0$. The origin is an attractor and for $x>0$ the trajectory moves to the left toward the origin and similarly for $x<0$ the trajectory also evolves toward the origin but along the increasing $x$ (See Fig. 1 (c)).

- Case IV, $c_{+} B C_{+}^{-1}+b<0$ and $c_{-} B C_{-}^{-1}+b>0$. The origin is a saddle and for $x>0$ the trajectory moves toward the origin, and for $x<0$ the trajectory evolves away from the origin (See Fig. 1 (d)).

Note that cases (c) and (d) in Fig. 1 can be thought of as (a) and (b), but with the time reversed.

We will now investigate the qualitative dynamics of (28) and (32) about the origin pertaining to the above four cases for $\varepsilon>0$. To this aim we first determine where lie the equilibrium points of (28) and (32).

To leading order we find

$$
\begin{aligned}
& \left(x_{+}^{*}, y_{+}^{*}\right)=\left(\frac{-C_{+}^{-1} B d \varepsilon}{c_{+} C_{+}^{-1} B+b}, \frac{-d \varepsilon}{c_{+} C_{+}^{-1} B+b}\right), \\
& \left(x_{-}^{*}, y_{-}^{*}\right)=\left(\frac{-C_{-}^{-1} B d \varepsilon}{c_{-} C_{-}^{-1} B+b}, \frac{-d \varepsilon}{c_{-} C_{-}^{-1} B+b}\right) .
\end{aligned}
$$

The equilibrium $\left(x_{+}^{*}, y_{+}^{*}\right)$ is an admissible equilibrium of our system if it exists for $x>0$ otherwise it is a virtual equilibrium. Similarly $\left(x_{-}^{*}, y_{-}^{*}\right)$ is an admissible equilibrium of our system if it exists for $x<0$ otherwise it is a virtual equilibrium. Switching $\varepsilon$ to a positive value implies birth of two equilibrium points, and this corresponds to so-called boundary-equilibrium bifurcations in non-smooth systems ${ }^{10}$.

In principle there is a possibility that switching $\varepsilon$ to a positive value gives rise to three distinct qualitative dynamics for each of the four cases enumerated in the previous section. Namely, we would expect to observe:

- birth of two admissible equilibrium points;

- birth of two virtual equilibrium points;

- birth of an admissible and a virtual equilibrium.

It would appear that twelve distinct scenarios are possible. However, the number of existing scenarios will be less then that and it is limited by the fact that at the points of intersection of $\mathcal{M}_{+}^{\varepsilon}$ and $\mathcal{M}_{-}^{\varepsilon}$ with $\Sigma$ the vector fields have the same sign. In other words we will not encounter small amplitude oscillations between $\mathcal{M}_{+}^{\varepsilon}$ and $\mathcal{M}_{-}^{\varepsilon}$ in the neighbourhood of the origin, and the flow will
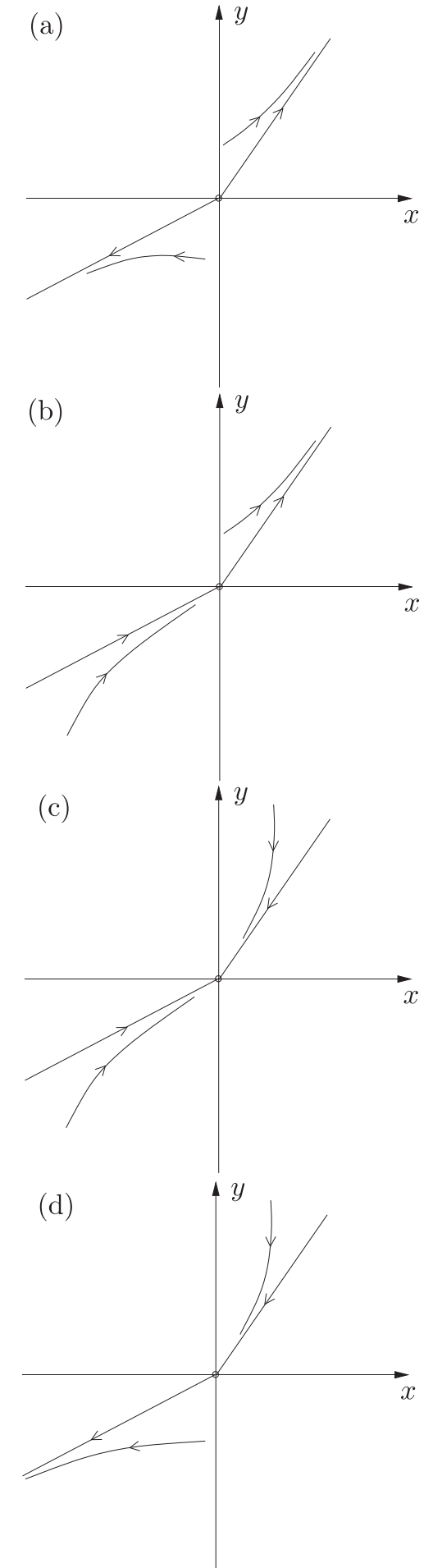

FIG. 1. Phase portraits of the reduced system (28) and (32) about the origin for $\varepsilon=0$; (a) Case I with the origin being a repeller of the reduced system, (b) Case II with the origin a saddle node of the reduced system, (c) Case III with the origin an attractor of the reduced system, and (d) Case IV with the origin a saddle node of the reduced system. 


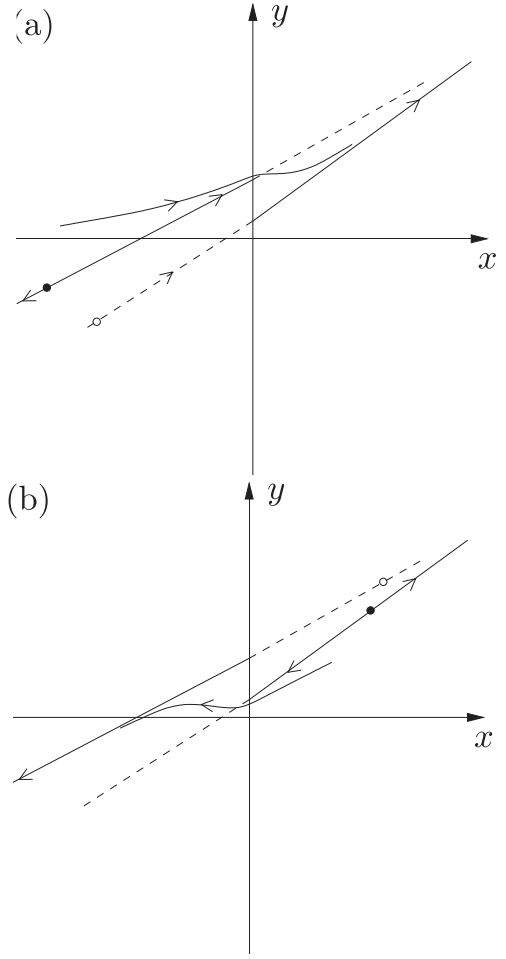

FIG. 2. Phase portraits of the slow-fast system (28) and (32) about the origin for $\varepsilon>0$ in the case when there exist (a) an admissible fixed point of $f_{-}$and $g_{-}$(black dot), and a virtual fixed point of $f_{+}, g_{+}$(small circle), and (b) an admissible fixed point of $f_{+}$and $g_{+}$(black dot), and a virtual fixed point of $f_{-}, g_{-}$(small circle).

either cross the switching manifold and diverge from the origin or it will reach an equilibrium point existing in the $\mathcal{O}(\varepsilon)$ neighbourhood of the origin.

To show this let us calculate the $(\dot{x}, \dot{y})$ on the slow manifolds about the origin for vector fields $\left(f_{+}, g_{+}\right)$, and $\left(f_{-}, g_{-}\right)$respectively. If it can be shown that $\dot{x}$ and $\dot{y}$ can take the opposite signs on the slow manifolds in some small neighbourhood of the origin then there is a possibility of oscillations across $\Sigma$. About the origin $\dot{y}_{ \pm}=$ $\varepsilon d$ and $\dot{x}_{ \pm}=B C_{ \pm}^{-1} \varepsilon d$. Therefore, both $\dot{y}_{ \pm}$and $\dot{x}_{ \pm}$are characterized by the same sign on $\Sigma$ and hence small scale oscillations across $\Sigma$ cannot occur.

\section{Phase portraits for Case I $\left(c_{+} B C_{+}^{-1}+b>0\right.$ and} $c_{-} B C_{-}^{-1}+b>0$ )

In the current case there are two scenarios possible: (i) for $d>0$ there exist an admissible fixed point of $f_{-}, g_{-}$ and a virtual fixed point of $f_{+}, g_{+}$(see Fig. 2(a)) or (ii) for $d<0$ there exist an admissible fixed point of $f_{+}$and $g_{+}$and a virtual fixed point of $f_{-}, g_{-}$(see Fig. 2(b)). Note that this follows from (33) under our assumption that $B>0$. In the former of these two cases the flow moves to the right across $\Sigma$ and in the latter to the left across $\Sigma$.
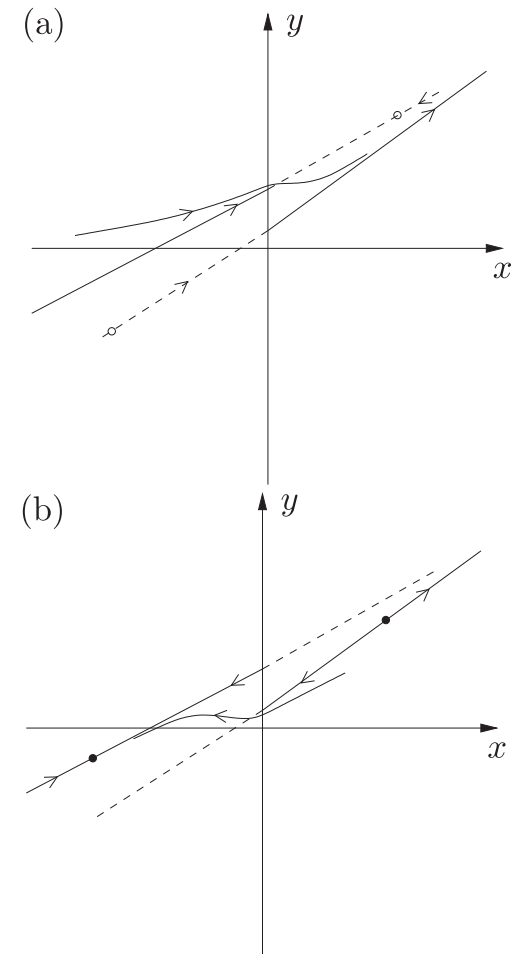

FIG. 3. Phase portraits of the slow-fast system (28) and (32) about the origin for $\varepsilon>0$ in the case when there exist (a) no admissible fixed points of $f_{-}, g_{-}$and $f_{+}, g_{+}$, and (b) there exist an admissible fixed point of $f_{+}, g_{+}$and of $f_{-}, g_{-}$(an attracting fixed point on the left and a saddle point on the right).

\section{Phase portraits for Case II $\left(c_{+} B C_{+}^{-1}+b>0\right.$ and $c_{-} B C_{-}^{-1}+b<0$ )}

In the current case there are two scenarios possible: (i) the existence of two virtual fixed points of $f_{-}, g_{-}$and of $f_{+}, g_{+}$for $d>0$ (see Fig. 3(a)) or (ii) there exist two admissible fixed points, one of $f_{+}, g_{+}$and the other of $f_{-}, g_{-}$for $d<0$ (see Fig. 3(b)). In the former of these two cases the flow moves to the right across $\Sigma$ and in the latter there exist an attracting fixed point on $\mathcal{M}_{-}^{\varepsilon}$ and a saddle point on $\mathcal{M}_{+}^{\varepsilon}$.

Cases III and IV can be easily obtained by considering phase portraits in Fig. 2 and Fig. 3 in reversed time. However, since these are used later in Sec. V we will present them for the sake of completeness.

\section{Phase portraits for Case III $\left(c_{+} B C_{+}^{-1}+b<0\right.$ and} $c_{-} B C_{-}^{-1}+b<0$ )

In the current case there are two scenarios possible: (i) the existence of an admissible fixed point of $f_{-}, g_{-}$and of a virtual fixed point of $f_{+}, g_{+}$for $d<0$ (see Fig. 4(a)) or (ii) there exist an admissible fixed points of $f_{+}, g_{+}$and a virtual fixed point of $f_{-}, g_{-}$for $d>0$ (see Fig. 4(b)). In 


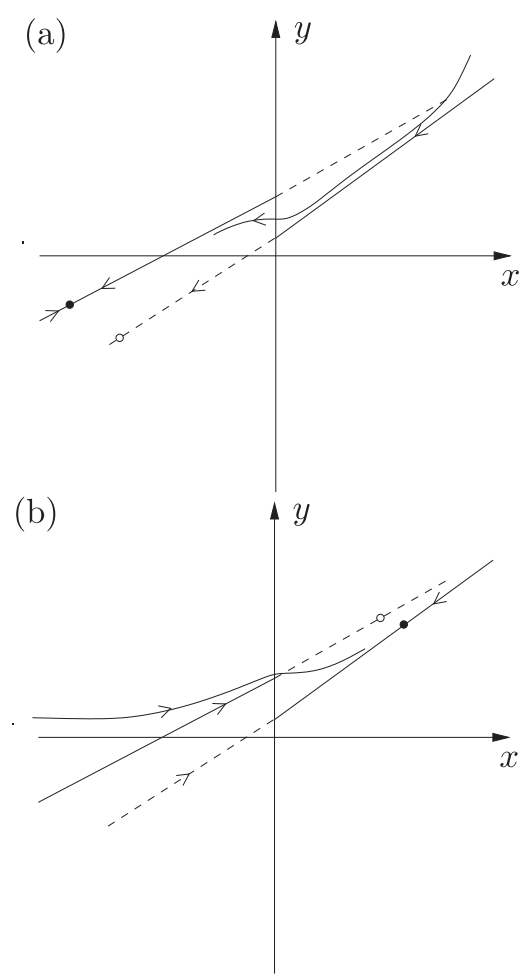

FIG. 4. Phase portraits of the slow-fast system (28) and (32) about the origin for $\varepsilon>0$ in the case when there exist (a) an admissible fixed points of $f_{-}, g_{-}$and a virtual fixed point of $f_{+}, g_{+}$, and (b) an admissible fixed point of $f_{+}, g_{+}$and a virtual fixed point of $f_{-}, g_{-}$.

either case the admissible fixed point is a local attractor. In the former of these two cases the flow moves to the left and in the latter to the right across $\Sigma$.

Phase portraits for Case IV $\left(c_{+} B C_{+}^{-1}+b<0\right.$ and $c_{-} B C_{-}^{-1}+b>0$ )

This case is equivalent to case II. Namely, for $d>0$ there exist an attracting fixed point on the right and a saddle point on the left of the switching manifold and the flow, for points sufficiently close to the switching surface, moves to the right across $\Sigma$; or for $d<0$ there are only virtual fixed points in the neighbourhood of the origin and the flow moves to the left across $\Sigma$

\section{EXAMPLE: BOX MODEL OF THERMOHALINE CIRCULATION}

In the following section we will apply our results to analyze a mathematical model of thermohaline circulation. The thermohaline circulation (or 'conveyor belt') in the ocean is a current which transports warm water near the surface from equatorial to polar regions, and cold water at deeper levels back from the polar regions to the equator. It is driven by heat (the warmer water

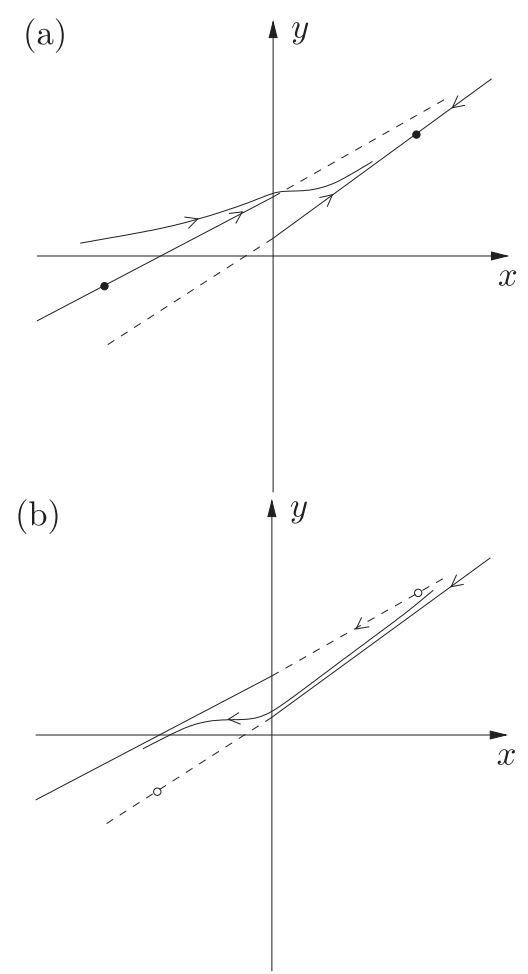

FIG. 5. Phase portraits of the slow-fast system (28) and (32) about the origin for $\varepsilon>0$ in the case when there exist (a) an admissible fixed points of $f_{-}, g_{-}$and $f_{+}, g_{+}$(an attracting fixed point on the right and a saddle point on the left), and (b) only virtual fixed points of $f_{+}, g_{+}$and of $f_{-}, g_{-}$.

cools and then due to greater density sinks as it reaches the poles) and salt (surface water becomes more salty at the equator as water is removed by evaporation due to the greater heat) which makes it heavier and allows less salty water to rise). Thermohaline circulation is a major source of heat transfer, and it has been disrupted in the past, leading to major climate change, and it may be that the effects of current climate change could change the circulation pattern again. Investigation of this has led back to the 'box' models of circulation developed by Stommel $^{16}$. In these models the polar region is described by one well-mixed box with temperature $T_{p}$ and salinity $S_{p}$, whilst the equatorial region is represented by another well-mixed box with temperature $T_{e}$ and salinity $S_{e}$ as shown in Figure 6. These boxes are connected near the surface and at depth by tubes which allow a flux $q$ to flow between the boxes which depends upon the temperature and salinity differences between the boxes. Stommel ${ }^{16}$ made the ansatz that the flux is dominated by the density difference between the regions,

$$
q=\gamma\left(\frac{\rho_{p}-\rho_{e}}{\rho_{0}}\right),
$$

for some constant $\gamma>0$, where $\rho_{p}$ and $\rho_{e}$ are the polar and equatorial densities of the water, given in terms of reference temperatures, salinity and density $T_{0}, S_{0}$ and 


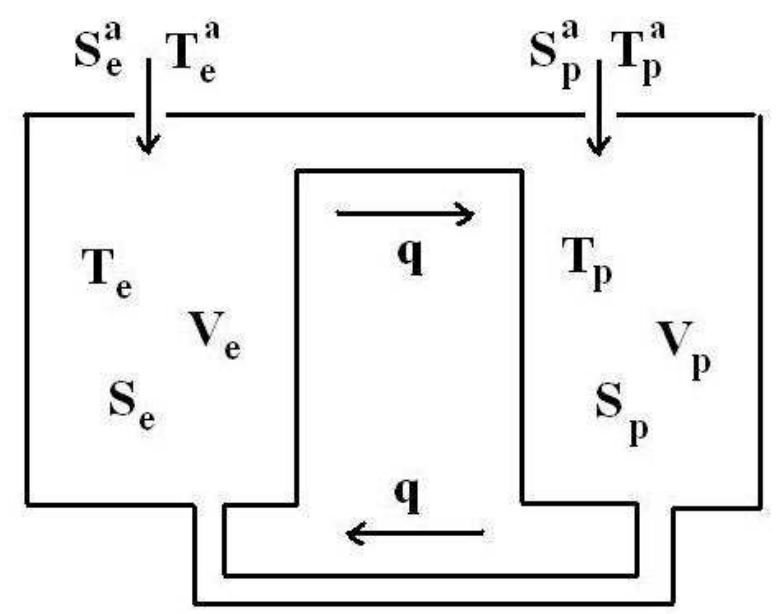

FIG. 6. Schematic diagram of the box model. Variables with subscripts $e$ are in the low latitude (equatorial) region and variables with subscripts $p$ are in the higher latitude (polar) region (after Dijkstra ${ }^{15}$ ).

$\rho_{0}$ by

$$
\rho=\rho_{0}\left(1-\alpha_{T}\left(T-T_{0}\right)+\alpha_{S}\left(S-S_{0}\right)\right),
$$

where $\alpha_{T}$ and $\alpha_{S}$ are thermal expansion and saline contraction coefficients. Thus

$$
q=\gamma\left(\alpha_{T}\left(T_{e}-T_{p}\right)-\alpha_{S}\left(S_{e}-S_{p}\right)\right)
$$

Following Stommel and Dijkstra ${ }^{15}$, assume that heat is added to the polar (resp. equatorial) box at a rate $T_{p}^{a}$ (resp. $T_{e}^{a}$ ) from the atmosphere, with $T_{e}^{a}-T_{p}^{a}>0$, and that salinity is increased at the equator (evaporation) and decreased at the pole (precipitation) at rates $S_{e}^{a}$ and $S_{p}^{a}$ with $S_{e}^{a}-S_{p}^{a}>0$. Then the model is defined by four differential equations:

$$
\begin{aligned}
V_{e} \frac{d}{d t} T_{e} & =C_{e}^{T}\left(T_{e}^{a}-T_{e}\right)+|q|\left(T_{p}-T_{e}\right), \\
V_{p} \frac{d}{d t} T_{e} & =C_{p}^{T}\left(T_{p}^{a}-T_{p}\right)+|q|\left(T_{e}-T_{p}\right), \\
V_{e} \frac{d}{d t} S_{e} & =C_{e}^{S}\left(S_{e}^{a}-S_{e}\right)+|q|\left(S_{p}-S_{e}\right), \\
V_{p} \frac{d}{d t} S_{e} & =C_{p}^{S}\left(S_{p}^{a}-S_{p}\right)+|q|\left(S_{e}-S_{p}\right),
\end{aligned}
$$

where $C_{e, p}^{T, S}$ are relaxation rates and $V_{e, p}$ are the volumes of the respective boxes. The quantity $q$ appears as a modulus since the direction of the flow does not change the dynamics in well-mixed boxes. It is standard to assume that the relaxation rates for the temperature are equal and constant, so $C_{e}^{T} / V_{e}=C_{p}^{T} / V_{p}=R_{T}$ and similarly for salinity $C_{e}^{S} / V_{e}=C_{p}^{S} / V_{p}=R_{S}$. With this simplifying assumption the equations can be combined to obtain two differential equations for the temperature difference $\Delta T=T_{e}-T_{p}$, and the salinity difference $\Delta S=S_{e}-S_{p}$ as

$$
\begin{aligned}
\frac{d}{d t} \Delta T & =R_{T}\left(\left[T_{e}^{a}-T_{a}^{p}\right]-\Delta T\right)-2|Q| \Delta T \\
\frac{d}{d t} \Delta S & =R_{S}\left(\left[S_{e}^{a}-S_{a}^{p}\right]-\Delta S\right)-2|Q| \Delta S
\end{aligned},
$$

where

$$
Q=\gamma\left(\alpha_{T} \Delta T-\alpha_{S} \Delta S\right) .
$$

Now define $\Delta T^{a}=T_{e}^{a}-T_{p}^{a}$ and $\Delta S^{a}=S_{e}^{a}-S_{p}^{a}$, and rescale the equations by setting

$$
x=\frac{\Delta T}{\Delta T^{a}}, y=\frac{\alpha_{S} \Delta S}{\alpha_{T} \Delta T^{a}}, \tau=R_{S} t
$$

(this is a slightly different scaling than that used in ${ }^{15}$, and will make it possible to use singular perturbation theory a little later; the same idea is used by Berglund and Gentz ${ }^{17}$ on a slightly different model) giving

$$
\begin{aligned}
\frac{d}{d \tau} x & =\frac{R_{T}}{R_{S}}(1-x)-A|x-y| x, \\
\frac{d}{d \tau} y & =\mu-(1+A|x-y|) y,
\end{aligned}
$$

where

$$
\mu=\frac{\alpha_{S} \Delta S^{a}}{\alpha_{T} \Delta T^{a}}, \quad A=\frac{2 \gamma \alpha_{T} \Delta T^{a}}{R_{S}} .
$$

To understand the behaviour of this system make one further (reasonable) assumption:

$$
R_{S} \ll R_{T}
$$

so that $\varepsilon=R_{S} / R_{T}$ is a small parameter (saline transport is slower than thermal transport). Then (39) can be rewritten as

$$
\begin{aligned}
\varepsilon \dot{x} & =(1-x)-\varepsilon A|x-y| x \\
\dot{y} & =\mu-(1+A|x-y|) y
\end{aligned}
$$

where the dot denotes differentiation with respect to $\tau$.

Equation (42) is almost in the form analyzed in previous sections, but to be able to apply the results of Sec. IV we will make a further transformation to bring the switching surface to the coordinate axis: set

$$
u=x-y, \quad v=1-y
$$

so $y=1-v$ and $x=1-v+u$. In terms of these variables (42) becomes

$$
\begin{aligned}
\varepsilon \dot{u} & =v-u+\varepsilon(1-\mu-v-A|u| u) \\
\dot{v} & =1-\mu-v+A|u|(1-v)
\end{aligned}
$$

This is now precisely the form considered in Sec. IV A with $C_{ \pm}=1, B=1$ and $D=1-\mu$.

Using equations (29) and (30) we can compute $u_{ \pm}(v)$ to leading order by noting that $a=g(0,0, \varepsilon)=\dot{v}(0,0, \varepsilon)=$ $1-\mu$. Thus we obtain $u_{ \pm}(v)=v-\varepsilon(1-\mu)+\varepsilon(1-$ $\mu)=v$. Finally, we compute the direction of the flow at 
the two intersection points of the slow manifold with the switching surface.

In our case, to leading order, we find $\dot{u}_{ \pm}^{i}=\dot{v}_{ \pm}^{i}$ and $\dot{u}_{ \pm}^{i}=1-\mu$. If $1-\mu$ is positive then the flow across the switching surface is to the right and upwards along the increasing values of $u$. Otherwise it is to the left and downwards along the decreasing values of $u$.

We have to consider now the final scenario which occurs when $\mu-1=0$. This is the case presented in Sec. IV B. For $\mu-1=0$ the reduced system (43), as well as the full system with non-zero $\varepsilon$, exhibit an equilibrium on the switching surface. Depending on the value of $A$ this equilibrium can be either:

- for $A \in(-1,1)$ a stable fixed point;

- for $A \in(-\infty,-1) \cup(1, \infty)$ a saddle point.

Since the position of the equilibrium is independent of $\varepsilon$ when $\mu=1$ then there is no change in qualitative dynamics when the full system is considered.

However, we may consider small size perturbations of $\mu-1$ about $\mu=1$ to determine the phase portraits of the slow-fast system (43). In this setting we can use the analysis from Sec. IVB with $d \varepsilon=1-\mu$ in equation (32). Then in the nomenclature of Sec. IV B we have to check the conditions on the signs of $c_{+} B C_{+}^{-1}+b$ and $c_{-} B C_{-}^{-1}+b$. We have that $c_{+}=A, c_{-}=-A, b=-1$, $C_{ \pm}=1$ and $B=1$. Therefore our conditions simplify to determining the signs of $A-1$ and $-A-1$. Clearly, depending on $A$, different scenarios, as enumerated in Sec. IV B may take place:

1. if $-1<A<1$, then $A-1<0$ and $-A-1<0$, and we have two possible scenarios as depicted in Fig. 4. If $1-\mu>0$ then the system crosses the switching surface along the increasing values of $u$ and tends towards the attractor on $u_{+}(v)$ which corresponds to the case presented in Fig. 4(b). On the other hand, if $1-\mu<0$, then the system crosses the switching surface along the decreasing values of $u$ and tends towards the attractor on $u_{-}(v)$, which corresponds to the case presented in Fig. 4(a);

2. if $-\infty<A<-1$, then $A-1<0$ and $-A-1>0$, and we have two possible scenarios as depicted in Fig. 5. If $1-\mu>0$ then there exists and attracting fixed point on $u_{+}$and an unstable fixed point on $u_{-}$, see Fig. 5(a). On the other hand, if $1-\mu<0$, then there no equilibrium points in some small neighbourhood of the origin and the flow moves across the switching surface, see Fig. 5(b);

3. if $1<A<\infty, A-1>0$ and $-A-1<0$, and we have two possible scenarios as depicted in Fig. 3. If $1-\mu>0$ there are no fixed points in some small neighbourhood of the origin, see Fig. 3(a), and if $1-\mu<0$ then there exists and attracting fixed point on $u_{-}$and an unstable fixed point on $u_{+}$, see Fig. $3(\mathrm{~b})$.
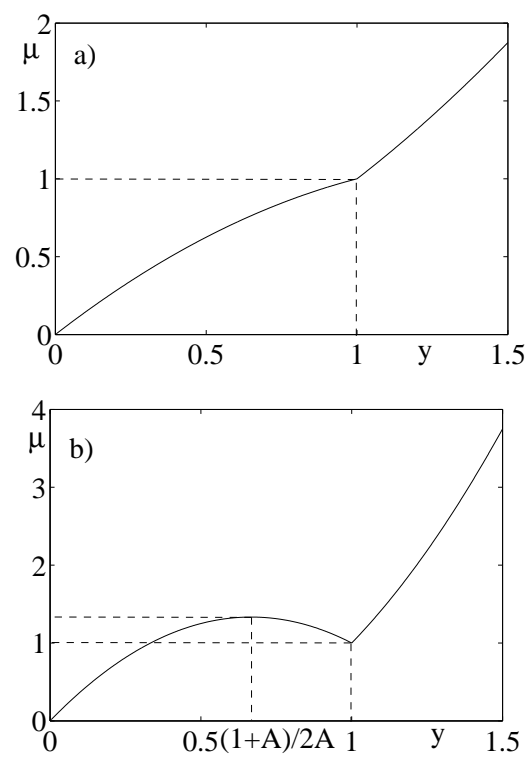

FIG. 7. Sketch of the graphs of the right hand side of (44) for (a) $A<1$; and (b) $A>1$.

The scenarios occurring under the variation of $\mu$ through 1 for $A \in(-\infty, 1) \cup(1, \infty)$ correspond to the non-smooth equivalent of a saddle-node bifurcation.

Having established the dynamics of the system across the switching manifolds for different values of $\mu$ and $A$ we can complete the investigations of our system by considering other invariant sets existing away from the switching manifold. Since the remaining analysis is standard and we wish to restrict ourselves to parameter values coming from our modelling we return to (42) to complete the analysis. In what follows we consider positive $A$ only; these values of $A$ are feasible physically - see (40). We first look at the existence of fixed points on the slow manifold $x=1$ (this is an approximate expression for the slow manifold up to order $\mathcal{O}(\varepsilon)$ ) of $(42)$. That is we will analyze

$$
\mu=\left\{\begin{array}{l}
(1+A) y-A y^{2} \text { for } y<1 \\
(1-A) y+A y^{2} \text { for } y>1
\end{array}\right.
$$

If $0<A<1$ then the right hand side of (42) is a monotonic increasing function with a discontinuity in the derivative at $y=1$ (see Fig. 7 a)), so for each value of $\mu$ there is a corresponding fixed point which is stable.

The case $A>1$ is more interesting. In this case the turning point of the quadratic defined in $y<1$ is $y=$ $(1+A) / 2 A$, where the function takes its maximum value $\mu=(1+A)^{2} / 4 A$. Since this turning point now lies in $y<1$, the graph of (44) has a turning point in $y<1$ and the decreasing branch attaches to the increasing branch of the parabola in $y>1$ at $y=1$, at which the right hand side of (44) takes the value of unity. Thus there are three cases (imagine moving a horizontal line of constant $\mu$ up or down in Figure 7 b)): 


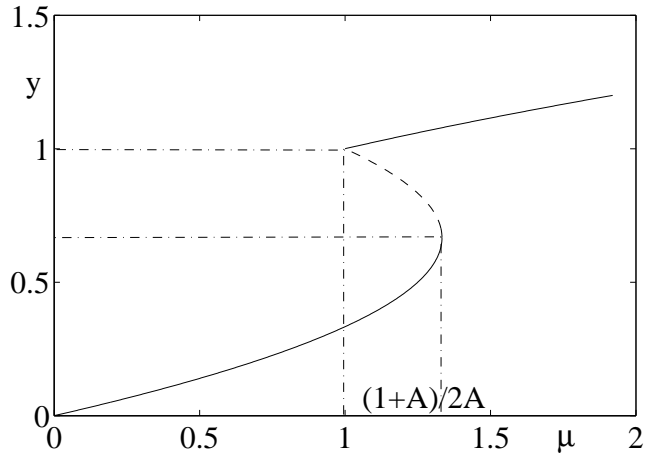

FIG. 8. Fixed points of system (42) on the slow manifold $x=$ 1 approximated to $\mathcal{O}(\varepsilon)$ for $A>1$. Note the existence of three equilibrium states for $1>\mu>(1+A) / 2 A$ which is the region of bi-stability. The dashed line denotes the unstable and the solid line the stable equilibrium points, and the dash-dotted lines denote the points where the saddle-node bifurcations occur.

- if $\mu<1$ the system has one stable solution in $y<1$;

- if $1<\mu<(1+A)^{2} / 4 A$ there are three solutions: two of these are stable, one in $y<1$ and the other in $y>1$;

- if $\mu>1$ then there is a single solution with $y>1$.

The bifurcation at $\mu=1$ is a non-smooth version of the saddle-node bifurcation, creating a pair of stable and unstable solutions and it has been discussed in detail in the $(u, v)$ co-ordinates (see cases 2 and 3 on the previous page); the bifurcation at $\mu=(1+A)^{2} / 4 A$ is a standard saddle-node bifurcation. The stability diagram is usually described as in Figure 8, which is essentially Figure 7(b) turned on its side, and shows the evolution of the fixed points as a function of $\mu$. Current estimations show that the ocean parameters are such that we lie on the upper branch of the stable solutions in Figure 8. This suggests two sources for concern: if the parameters are in the (middle) bistable region, then a perturbation of initial conditions, i.e. some extraneous effect not described by the box model, could push us onto the weaker lower stable solution; or the parameter $\mu$ may be changing in such a way that it approaches $\mu=(1+A)^{2} / 4 A$ at which point the system moves dramatically to the stable lower solution.

Note that the interpretation of solutions relies on the direction of the flow $q$ in (34), and in terms of the variables $x$ and $y$ defined in (38) this becomes proportional to $x-y$, i.e. to $1-y$ on the slow manifold. The concern for the implications of climate change is that if $A>1$ and $1<\mu<(1+A)^{2} /(4 A)$, a stable solution with $q>0$ can coexist with a stable solution with $q<0$, indicating the possibility of flow reversal.

\section{CONCLUSIONS}

We consider the effects of singular perturbations on the dynamics of planar piecewise-smooth systems with discontinuous jacobians and an equilibrium point on the switching surface. We describe phase space topology of planar systems: the slow and fast dynamics being onedimensional. The slow manifold of the full system exhibits $\mathcal{O}(\varepsilon)$ discontinuity across the switching manifold. In the case that the vector field is transversal across the switching surface, in spite of the presence of the discontinuity across the switching surface, small scale boundary oscillations between the slow manifolds are not possible. This is a standard result, and it can be shown using Fenichel's ${ }^{12}$ theory.

The main purpose of the paper is the investigation of the qualitative dynamics when the slow system has an equilibrium on the switching surface. Four distinct dynamic scenarios are found. This analysis is then used to investigate the dynamics of a planar piecewise-smooth slow-fast box model of thermohaline circulation. Combined effects of discontinuous nonlinearity and fast dynamics give rise to a boundary-equilibrium bifurcation of a fold type.

The four scenarios observed in planar slow-fast systems will be observed in higher dimensions (with $n$-dimensional slow dynamics), and it is very likely that additional dynamics will arise as well; boundaryequilibrium bifurcations in three-dimensional piecewisesmooth flows lead, for instance, to a non-smooth equivalent of a Hopf bifurcation with a limit cycle growing linearly in amplitude from an equilibrium colliding with the switching manifold ${ }^{18}$. This rises a question whether additional dynamics can be triggered by the presence of more than one fast dimension.

Another open issue is the effect of singular perturbations on grazing bifurcations. In the case when a planar Filippov type system exhibits so-called grazing-sliding scenario a singular perturbation applied to a system may result in the onset of micro-chaotic oscillations ${ }^{14}$. Hence grazing-sliding is not robust against singular perturbations. The question now arises if this would also be the case when grazing occurs in the absence of sliding which is a co-dimension one event in the class of systems considered here.

${ }^{1}$ S. Banerjee and G. Verghese. Nonlinear Phenomena in Power Electronics. IEEE press, New York, 2001.

${ }^{2}$ M. I. Feigin. Forced Oscillations in Systems With Discontinuous Nonlinearities. Nauka, Moscow, 1994. In Russian.

${ }^{3}$ K. Popp, N. Hinrichs, and M. Oestreich. Dynamical behaviour of friction oscillators with simultaneous self and external excitation. Sadhana (Indian Academy of Sciences), 20:627-654, 1995.

${ }^{4}$ U. Galvanetto and S. R. Bishop. Dynamics of a simple damped oscillator undergoing stick-slip vibrations. Meccanica, 34:337$347,2000$.

${ }^{5}$ F. Peterka. Part 1: Theoretical analysis of $n$-multiple $(1 / n)$ impact solutions. CSAV Acta Technica, 19:462-473, 1974.

${ }^{6}$ C. Budd, K.A. Cliffe, and F. Dux. The effect of frequency and clearance varions on one-degree of freedom impact oscillators. $J$. Sound E Vibration, 184, 1995. 
${ }^{7}$ M. Oestreich, N. Hinrichs, K. Popp, and C. J. Budd. Analytical and experimental investigation of an impact oscillator. Proceedings of the ASME 16th Biennal Conf. on Mech. Vibrations and Noise, 1996.

${ }^{8}$ R.I. Leine. Bifurcations in Discontinuous Mechanical Systems of Filippov-Type. PhD thesis, Teknische Universiteit Eindhoven, The Netherlands, 2000.

${ }^{9}$ R.I. Leine and H. Nijmeijer. Dynamics and Bifurcations of NonSmooth Mechanical Systems, volume 18 of Lecture Notes in Applied and Computational Mechanics. Springer-Verlag, Berlin Heidelberg, 2004.

${ }^{10}$ M. di Bernardo, C. J. Budd, A. R. Champneys, and P. Kowalczyk. Piecewise-smooth Dynamical Systems: Theory and Applications. Springer-Verlag, 2008.

${ }^{11} \mathrm{~S}$. A. Lomov. Introduction to the General Theory of Singular Perturbations. American Mathematical Society, 1992.

${ }^{12}$ N. Fenichel. Geometric singular perturbation theory for ordinary differential equations. Journal of Differential Equations, 31:5398, 1979.

${ }^{13}$ L. M. Fridman. Slow periodic motions with internal sliding modes in variable structire systems. International Journal of Control,
75:524-537, 2002.

${ }^{14}$ J. Sieber and P. Kowalczyk. Small-scale instabilities in dynamical systems with sliding. Physica D, 239(1-2):44-57, 2010.

${ }^{15}$ H. A. Dijkstra. Nonlinear Physical Oceanography. Springer, Dordrecht, 2005.

${ }^{16}$ H. M. Stommel. Thermohaline convection with two stable regimes of flow. Tellus, 13:79-89, 1961.

${ }^{17}$ N. Berglund and B. Gentz. Metastability in simple climate models: pathwise analysis of slowly driven langevin equations. Stochastics and Dynamics, 2:327-356, 2002.

${ }^{18}$ M. di Bernardo, A. Nordmark, and G. Olivar. Discontinuityinduced bifurcations of equlibria in piecewise-smooth and impacting dynamical systems. Physica D, 237:119-136, 2008.

${ }^{19}$ L. M. Fridman. Slow periodic motions with internal sliding modes in variable structure systems. Int. J. of Control, 75:524-527, 2002

\section{Acknowledgements}

Research partially funded by EPSRC grant EP/E050441/1 and the University of Manchester. 